Bulletin of the Section of Logic

Volume 49/4 (2020), pp. 359-375

http://dx.doi.org/10.18778/0138-0680.2020.13

Satoru Niki

\title{
EMPIRICAL NEGATION, CO-NEGATION AND THE CONTRAPOSITION RULE II: PROOF-THEORETICAL INVESTIGATIONS
}

\begin{abstract}
We continue the investigation of the first paper where we studied logics with various negations including empirical negation and co-negation. We established how such logics can be treated uniformly with R. Sylvan's $\mathbf{C C}_{\omega}$ as the basis. In this paper we use this result to obtain cut-free labelled sequent calculi for the logics.
\end{abstract}

Keywords: Empirical negation, co-negation, labelled sequent calculus, intuitionism.

\section{Introduction}

In the first paper, we semantically investigated how some logics with nonstandard negation (IPC $\sim$ [1, 2], $\mathbf{T C C} \omega$ [4], daC [9] and $\mathbf{C C}_{\omega}$ [10]) are related to each other. In particular, we noted how the difference between IPC $^{\sim}$ and $\mathbf{T} \mathbf{C C} \omega$ can be understood as the difference between Kripke and Beth semantics. We also observed, by giving a uniform axiomatisation, how other logics can be captured in the semantics of $\mathbf{C C}_{\omega}$ in terms of frame conditions. These frames conditions will play an essential role in the proof-theoretical investigation of the present paper.

In this paper, we first re-introduce some definitions and results from the first paper. Then we formulate labelled sequent calculi for the logics, and

Presented by: Andrzej Indrzejczak

Received: April 18, 2020

Published online: August 15, 2020

(C) Copyright for this edition by Uniwersytet Łódzki, Łódź 2020 
show cut-admissibility and the equivalence of the calculi with Hilbert-style formulations. Finally we have discussions about some topics related to the contents of the two papers.

\section{Preliminaries}

Let us use the following notations for metavariables.

- $p, q, r, \ldots$ for propositional variables.

- $A, B, C, \ldots$ for formulae.

In this paper, we shall consider the following propositional language

$$
\mathcal{L}::=p|(A \wedge B)|(A \vee B)|(A \rightarrow B)| \sim A .
$$

Parentheses will be omitted if there is no fear of ambiguity. We shall use the convention $A \leftrightarrow B:=(A \rightarrow B) \wedge(B \rightarrow A)$.

We shall give Hilbert-style proof systems for $\mathbf{C C}_{\omega}$, daC, $\mathbf{T} \mathbf{C C}_{\omega}$ and IPC $^{\sim}$, using the uniform axiomatisations we established in the first paper. We identify the systems with the logics themselves for convenience, and denote them simply as $\mathbf{C C}_{\omega}$, daC etc..

Definition 2.1. We will consider following axiom schemata and rules.

Axioms

[Ax1]

[Ax2]

[Ax3]

$[\mathrm{Ax} 4]$

[Ax5]

[Ax6]

[Ax7]

$[\mathrm{Ax} 8]$

[Ax9]

[Ax10]

[Ax11]

$$
\begin{aligned}
& A \rightarrow(B \rightarrow A) \\
&(A \rightarrow(B \rightarrow C)) \rightarrow((A \rightarrow B) \rightarrow(A \rightarrow C)) \\
&(A\wedge B) \rightarrow A \\
&(A\wedge B) \rightarrow B \\
&(C \rightarrow A) \rightarrow((C \rightarrow B) \rightarrow(C \rightarrow(A \wedge B))) \\
& A \rightarrow(A \vee B) \\
& B \rightarrow(A \vee B) \\
&(A \rightarrow C) \rightarrow((B \rightarrow C) \rightarrow((A \vee B) \rightarrow C)) \\
& A \vee \sim A \\
& \sim A \rightarrow(\sim \sim A \rightarrow B) \\
& \sim \sim A \rightarrow A
\end{aligned}
$$


$[\mathrm{Ax} 12]$

$[\mathrm{Ax} 13]$

$$
\begin{gathered}
\sim(\sim(A \vee B) \vee A) \rightarrow B \\
(\sim A \wedge \sim B) \rightarrow \sim(A \vee B)
\end{gathered}
$$

$\underline{\text { Rules }}$

$$
[\mathrm{MP}] \frac{A \quad A \rightarrow B}{B} \quad[\mathrm{RC}] \frac{A \rightarrow B}{\sim B \rightarrow \sim A}
$$

The logics $\mathbf{C C}_{\omega}$, daC, $\mathbf{T} \mathbf{C C}_{\omega}$ and $\mathbf{I P C}{ }^{\sim}$ are each defined by $[\mathrm{MP}]$, $[\mathrm{RC}]$ and the following axioms.

$$
\begin{aligned}
& \mathbf{C C}_{\omega}: {[\mathrm{Ax} 1]-[\mathrm{Ax} 9] \text { and }[\mathrm{Ax} 11] . } \\
& \text { daC }:[\mathrm{Ax} 1]-[\mathrm{Ax} 9] \text { and }[\mathrm{Ax} 12] . \\
& \text { TCC }_{\omega}:[\mathrm{Ax} 1]-[\mathrm{Ax} 10] . \\
& \text { IPC }^{\sim}:[\mathrm{Ax} 1]-[\mathrm{Ax} 10] \text { and }[\mathrm{Ax} 13] .
\end{aligned}
$$

The derivability in $\mathbf{C C}_{\omega}$ is denoted by $\vdash_{c}$. We recall that $\mathbf{I P C} \sim$ and $\mathbf{C C}_{\omega}$ are the strongest and the weakest of the four systems, and $\mathbf{d a C}$ and TCC $\omega$ lie somewhere between the two, while being mutually incomparable.

Semantically, the Kripke semantics of $\mathbf{C C}_{\omega}$ gives the basis for our inquiry.

Definition 2.2 (Semantics of $\mathbf{C C}_{\omega}$ ). A Kripke frame $\mathcal{F}_{\mathcal{K}}^{c}$ for $\mathbf{C C}_{\omega}$ is a triple $(W, \leq, S)$, where $S \subseteq W \times W$ is a reflexive and symmetric (accessibility) relation such that $u \leq v$ and $u S w$ implies $v S w$, i.e. $S$ is upward closed. A Kripke model $\mathcal{M}_{\mathcal{K}}^{c}$ for $\mathbf{C C}_{\omega}$ a pair $\left(\mathcal{F}_{\mathcal{K}}^{c}, \mathcal{V}\right)$, where $\mathcal{V}$ is a mapping that assigns a set of worlds $\mathcal{V}(p) \subseteq W$ to each propositional variable $p$. We assume $\mathcal{V}$ to be monotone, viz. $w \in \mathcal{V}(p)$ and $w^{\prime} \geq w$ implies $w^{\prime} \in \mathcal{V}(p)$. To denote a model, we shall use both $\mathcal{M}_{\mathcal{K}}^{c}$ and $\left(\mathcal{F}_{\mathcal{K}}^{c}, \mathcal{V}\right)$ interchangeably.

Given $\mathcal{M}_{\mathcal{K}}^{c}$, the forcing (or valuation) of a formula in a world, denoted $\mathcal{M}_{\mathcal{K}}^{c}, w \Vdash_{\mathcal{K}_{c}} A$, is inductively defined as follows.

$$
\begin{aligned}
& \mathcal{M}_{\mathcal{K}}^{c}, w \Vdash_{\mathcal{K}_{c}} p \quad \Longleftrightarrow w \in \mathcal{V}(p) . \\
& \mathcal{M}_{\mathcal{K}}^{c}, w \Vdash_{\mathcal{K}_{c}} A \wedge B \quad \Longleftrightarrow \mathcal{M}_{\mathcal{K}}^{c}, w \Vdash_{\mathcal{K}_{c}} A \text { and } \mathcal{M}_{\mathcal{K}}^{c}, w \Vdash_{\mathcal{K}_{c}} B \text {. } \\
& \mathcal{M}_{\mathcal{K}}^{c}, w \Vdash_{\mathcal{K}_{c}} A \vee B \quad \Longleftrightarrow \mathcal{M}_{\mathcal{K}}^{c}, w \Vdash_{\mathcal{K}_{c}} A \text { or } \mathcal{M}_{\mathcal{K}}^{c}, w \Vdash_{\mathcal{K}_{c}} B . \\
& \mathcal{M}_{\mathcal{K}}^{c}, w \Vdash_{\mathcal{K}_{c}} A \rightarrow B \quad \Longleftrightarrow \quad \text { for all } w^{\prime} \geq w \text {, if } \mathcal{M}_{\mathcal{K}}^{c}, w^{\prime} \Vdash_{\mathcal{K}_{c}} A \text {, } \\
& \text { then } \mathcal{M}_{\mathcal{K}}^{c}, w^{\prime} \Vdash_{\mathcal{K}_{c}} B \text {. } \\
& \mathcal{M}_{\mathcal{K}}^{c}, w \Vdash_{\mathcal{K}_{c}} \sim A \quad \Longleftrightarrow \mathcal{M}_{\mathcal{K}}^{c}, w^{\prime} \nVdash_{\mathcal{K}_{c}} A \text { for some } w^{\prime} \text { such that } w S w^{\prime} .
\end{aligned}
$$


We shall occasionally denote $u S v$ also by $v S^{-1} u$. As $S$ is symmetric in $\mathbf{C C}_{\omega}$, this distinction is not quite necessary. This however clarifies appeals to symmetry in proofs, which becomes significant in a broader context.

Now the following gives the summary of semantical characterisations for our logics.

TheOREM 2.3. Let $\mathcal{F}_{\mathcal{K}}^{c}$ be a $\mathbf{C C}_{\omega}$-frame. Then the following conditions are equivalent:

(i) $\mathbf{C C}_{\omega}$ is sound and weakly complete with respect to the class of all $\mathbf{C C}_{\omega}$-frames.

(ii) $\mathbf{d a C}$ is sound and weakly complete with respect to the class of $\mathbf{C C}_{\omega^{-}}$ frames satisfying the condition

$$
\forall u, v\left(u S v \text { implies } \exists w S^{-1} v(w \leq u \text { and } w \leq v)\right) .
$$

(iii) $\mathbf{T} \mathbf{C C}_{\omega}$ is sound and weakly complete with respect to $\mathbf{C C}_{\omega}$-frames where $S$ is transitive.

(iv) $\mathbf{I P C} \sim$ is sound and strongly complete with respect to $\mathbf{C C}_{\omega}$-frames where $S$ is transitive and satisfying the condition

$$
\forall u, v, w\left(u S v \text { and } u S w \text { implies } \exists x S^{-1} u(v \geq x \text { and } w \geq x) .\right.
$$

Proof: (i) and (iii) are established in [10] and [4], respectively. (ii) is Corollary 5.2 of the first paper, and (iv) is a consequence of Proposition 5.6 of the same paper, as discussed thereafter.

\section{Labelled sequent calculus}

In this section, we define a labelled sequent calculus for some of the logics we have treated $\left(\mathbf{C C}_{\omega}, \mathbf{T} \mathbf{C C}_{\omega}, \mathbf{d a C}, \mathbf{I P C} \sim\right)$, with the aid of the insights obtained in the last section regarding their relationship. We shall show the admissibility of cut and the correspondence with the Hilbert-style system.

A labelled formula is an expression of the form $x: A$, where $A$ is a formula and $x$ is a label. We shall use $x, y, z \ldots$ for labels. We shall additionally consider relational atoms, which either have the form $x S y$, or $x \leq y$. An item is either a labelled formula or a relational atom. We denote items by $\alpha, \beta, \ldots$ A sequent has the form $\Gamma \Rightarrow \Delta$, where $\Gamma$ and $\Delta$ are finite multisets of items. 
We shall consider the following calculus $\mathbf{G} 3 \mathbf{c c}_{\omega}$.

DeFinition $3.1\left(\mathbf{G 3 c c}_{\omega}\right)$.

$$
\begin{aligned}
& x \leq y, x: p, \Gamma \Rightarrow \Delta, y: p(\mathrm{Ax} 1) x \leq y, \Gamma \Rightarrow \Delta, x \leq y(\operatorname{Ax} 2) \\
& x S y, \Gamma \Rightarrow \Delta, x S y(\mathrm{Ax} 3) \\
& \frac{x: A, x: B, \Gamma \Rightarrow \Delta}{x: A \wedge B, \Gamma \Rightarrow \Delta}(\mathrm{L} \wedge) \quad \frac{\Gamma \Rightarrow \Delta, x: A \quad \Gamma \Rightarrow \Delta, x: B}{\Gamma \Rightarrow \Delta, x: A \wedge B}(\mathrm{R} \wedge) \\
& \frac{x: A, \Gamma \Rightarrow \Delta \quad x: B, \Gamma \Rightarrow \Delta}{x: A \vee B, \Gamma \Rightarrow \Delta}(\mathrm{L} \vee) \frac{\Gamma \Rightarrow \Delta, x: A, x: B}{\Gamma \Rightarrow \Delta, x: A \vee B}(\mathrm{R} \vee) \\
& \frac{x \leq y, x: A \rightarrow B, \Gamma \Rightarrow \Delta, y: A \quad x \leq y, x: A \rightarrow B, y: B, \Gamma \Rightarrow \Delta}{x \leq y, x: A \rightarrow B, \Gamma \Rightarrow \Delta}(\mathrm{L} \rightarrow) \\
& \frac{x \leq y^{*}, y^{*}: A, \Gamma \Rightarrow \Delta, y^{*}: B}{\Gamma \Rightarrow \Delta, x: A \rightarrow B}(\mathrm{R} \rightarrow) \\
& \frac{x S y^{*}, \Gamma \Rightarrow \Delta, y^{*}: A}{x: \sim A, \Gamma \Rightarrow \Delta}(\mathrm{L} \sim) \quad \frac{x S y, y: A, \Gamma \Rightarrow \Delta, x: \sim A}{x S y, \Gamma \Rightarrow \Delta, x: \sim A}(\mathrm{R} \sim) \\
& \frac{x \leq x, \Gamma \Rightarrow \Delta}{\Gamma \Rightarrow \Delta} \text { (Ref) } \quad \frac{x \leq z, x \leq y, y \leq z, \Gamma \Rightarrow \Delta}{x \leq y, y \leq z, \Gamma \Rightarrow \Delta} \text { (Trans) } \\
& \frac{x S x, \Gamma \Rightarrow \Delta}{\Gamma \Rightarrow \Delta}\left(\operatorname{Ref}_{S}\right) \quad \frac{x S y, y S x, \Gamma \Rightarrow \Delta}{x S y, \Gamma \Rightarrow \Delta}\left(\operatorname{Sym}_{S}\right) \\
& \frac{x \leq y, x S z, y S z, \Gamma \Rightarrow \Delta}{x \leq y, x S z, \Gamma \Rightarrow \Delta}(\mathrm{Up})
\end{aligned}
$$

A proof (derivation/deduction) of a sequent $\Gamma \Rightarrow \Delta$ in $\mathbf{G B C c}_{\omega}$ (to be denoted $\vdash_{G c} \Gamma \Rightarrow \Delta$ ) is a tree whose root is the sequent, whose nodes are applications of rules, and whose leaves are axioms (0-premise rules).

In the rules, variables indicated by * are eigenvariables, meaning that they cannot occur in the conclusion of the rules. $\Gamma, \Delta$ are called contexts, and non-context items in the conclusion are called principal. The calculus is in a large part an amalgamation of the labelled calculus for modal logic [6] and intuitionistic logic [7]. It has the rules $\left(\operatorname{Ref}_{S}\right)$ and $\left(\operatorname{Sym}_{S}\right)$ corre- 
sponding to the reflexivity and symmetry in $\mathbf{C C}_{\omega}$. Additionally, it has the rule (Up) corresponding to the condition for upward closure in $\mathbf{C C}_{\omega}$.

We shall also consider the following additional rules, corresponding to the additional frame conditions for $\mathbf{d a C}, \mathbf{T} \mathbf{C C}_{\omega}$ and $\mathbf{I P C}{ }^{\sim}$, to $\mathbf{G} \mathbf{3} \mathbf{c c}_{\omega}$.

DEFINITION 3.2.

$$
\begin{gathered}
\frac{x S y, y S z^{*}, z^{*} \leq x, z^{*} \leq y, \Gamma \Rightarrow \Delta}{x S y, \Gamma \Rightarrow \Delta}(\operatorname{Pr}) \frac{x S z, x S y, y S z, \Gamma \Rightarrow \Delta}{x S y, y S z, \Gamma \Rightarrow \Delta}\left(\operatorname{Trans}_{S}\right) \\
\frac{x S y, x S z, t^{*} \leq y, t^{*} \leq z, x S t^{*}, \Gamma \Rightarrow \Delta}{x S y, x S z, \Gamma \Rightarrow \Delta}(\mathrm{De})
\end{gathered}
$$

Where, as before, labels indicted with $*$ are eigenvariables. The intention is that the addition of (Pr) should correspond to $\mathbf{d a C},\left(\operatorname{Trans}_{S}\right)$ to $\mathbf{T} \mathbf{C C} \omega$, and $\left(\operatorname{Trans}_{S}\right)$ and (De) to $\mathbf{I P C} \mathbf{C}^{\sim}$. We shall denote the addition of some of these rules to $\mathbf{G} \mathbf{3} \mathbf{c c}_{\omega}$ by $\mathbf{G} \mathbf{3} \mathbf{c c}_{\omega}^{+}$, and the deducibility is denoted by $\vdash_{G c+}$.

We shall later observe how the sequent calculi correspond to the Hilbertstyle systems. We now proceed with checking some standard properties of the calculi.

Proposition 3.3. $\vdash_{G c} x \leq y, x: A, \Gamma \Rightarrow \Delta, y: A$

Proof: By [8, Lemma 12.25], we only have to consider the case for $\sim$. When $A \equiv \sim B$,

$$
\begin{aligned}
& \frac{z \leq z, z: B, x S z, x \leq y, y S z, \Gamma \Rightarrow \Delta, y: \sim B, z: B}{z: B, x S z, x \leq y, y S z, \Gamma \Rightarrow \Delta, y: \sim B, z: B} \text { (Ref) } \\
& \frac{z: B, x S z, x \leq y, y S z, \Gamma \Rightarrow \Delta, y: \sim B, z: B}{x S z, x \leq y, y S z, \Gamma \Rightarrow \Delta, y: \sim B, z: B}(\mathrm{R} \sim) \\
& \frac{x S z, x \leq y, \Gamma \Rightarrow \Delta, y: \sim B, z: B}{x \leq y, x: \sim B, \Gamma \Rightarrow \Delta, y: \sim B}(\mathrm{~L} \sim)
\end{aligned}
$$

where the first line is obtained from the inductive hypothesis.

Definition 3.4 (substitution of labels). We define the substitution of a label by another label $x[z / w]$, substitution for an item $\alpha[z / w]$ and for a multiset $\Gamma[z / w]$ by the following clauses. $(\circ \in\{\leq, S\})$

$$
\begin{aligned}
x[z / w] & \equiv w \text { if } x \equiv z . \\
x[z / w] & \equiv x \text { if } x \not \equiv z . \\
\alpha[z / w] & \equiv x[z / w] \circ y[z / w] \text { if } \alpha \equiv x \circ y . \\
\alpha[z / w] & \equiv x[z / w]: A \text { if } \alpha \equiv x: A . \\
\Gamma[z / w] & \equiv\{\alpha[z / w]: \alpha \in \Gamma\}
\end{aligned}
$$


We shall denote instances of substitution by (Sub). In addition, we shall write $\mathbf{G} 3 \mathbf{c c}_{\omega}^{+} \vdash_{n} \Gamma \Rightarrow \Delta$ if the sequent has a derivation whose depth is less than $n$. We say a rule is depth-preserving admissible (dp-admissible) if and only if: if there are derivations of the premises of the rule each with the depth less than $n$, then there exists a derivation of the conclusion with the depth less than $n$. If the depth is not preserved, we just say the rule is admissible. We shall indicate an application of an admissible rule by a dashed line.

Proposition 3.5 (dp-admissibility of substitution). The rule $\frac{\Gamma \Rightarrow \Delta}{\Gamma[z / w] \Rightarrow \Delta[z / w]}$ (Sub) is dp-admissible in $\mathbf{G 3 c c}_{\omega}^{+}$.

Proof: We argue by induction on the depth of deduction. The intuitionistic rules are already treated in [8, Lemma 12.26]. For others, the case for (Ax2) is immediate, since the result of the substitution is also an instance of $(\mathrm{Ax} 2)$. The case for $(\mathrm{L} \sim)$ and $(\mathrm{R} \sim)$ are similar to those of $\mathrm{R} \square$ and $\mathrm{L} \square$ in modal calculi, respectively; c.f. [8, Lemma 11.4]. The other rules are instances of either the scheme for mathematical rules or the geometric rule scheme $[8$, pp. 98, 134], so can be dealt with by the methodology of [8, Lemma 11.4].

We shall now move on to consider structural rules.

DEFinition 3.6 (structural rules).

$$
\begin{gathered}
\frac{\Gamma \Rightarrow \Delta}{\alpha, \Gamma \Rightarrow \Delta}(\mathrm{LW}) \quad \frac{\Gamma \Rightarrow \Delta}{\Gamma \Rightarrow \Delta, \alpha}(\mathrm{RW}) \\
\frac{\alpha, \alpha, \Gamma \Rightarrow \Delta}{\alpha, \Gamma \Rightarrow \Delta}(\mathrm{LC}) \frac{\Gamma \Rightarrow \Delta, \alpha, \alpha}{\Gamma \Rightarrow \Delta, \alpha}(\mathrm{RC}) \\
\frac{\Gamma \Rightarrow \Delta, \alpha \quad \alpha, \Gamma^{\prime} \Rightarrow \Delta^{\prime}}{\Gamma, \Gamma^{\prime} \Rightarrow \Delta, \Delta^{\prime}}(\mathrm{Cut})
\end{gathered}
$$

Our goal is to prove that (Cut) is admissible. For this purpose we check that the rules of Weakening (LW,RW) and Contraction (LC,RC) are dp-admissible. We start with Weakening.

Proposition 3.7 (dp-admissibility of Weakening). (LW) and (RW) are dp-admissible in $\mathbf{G 3 C c}_{\omega}^{+}$. 
Proof: The proof is by induction on the depth of deduction. In cases of applications of $(\mathrm{Ax} 1)-(\mathrm{Ax} 3)$, the result of Weakening is again an instance of the axiom. For other rules, we apply the inductive hypothesis to the premises of the rule, and thereafter apply the rule to obtain the desired sequent; however for rules involving eigenvariables, we first need to apply dp-admissible substitution (Proposition 3.5) to substitute the eigenvariable with a fresh variable, so as to avoid the clash of variables. Then we apply the above procedure.

For Contraction, we first need to demonstrate that the rules of $\mathbf{G} 3 \mathbf{c c}_{\omega}^{+}$ are $d p$-invertible: that is, given a derivation of the conclusion of a rule, we can find a depth-preserving derivation of the premises.

LEMMA 3.8. The rules of $\mathbf{G} \mathbf{3} \mathbf{c c}_{\omega}^{+}$are $d p$-invertible.

Proof: We argue by induction on the depth of deduction. For the intuitionistic rules, we refer to [8, Theorem 12.28]. For the rules $(\mathrm{R} \sim)$, (Up), $\left(\operatorname{Ref}_{S}\right),\left(\operatorname{Sym}_{S}\right),\left(\operatorname{Trans}_{S}\right),(\operatorname{Pr})$ and $(\mathrm{De})$, we can invert the sequent by dp-admissible weakening.

The case for $(\mathrm{L} \sim)$ is quite similar to that of $\mathrm{R} \square$ for modal logic [8, Lemma 11.7]. If $\vdash_{0} x: \sim A, \Gamma \Rightarrow \Delta$, then the derivation is an instance of $(\mathrm{Ax} 1),(\mathrm{Ax} 2)$ or $(\mathrm{Ax} 3)$. In each case, $x S y, \Gamma \Rightarrow \Delta, y: A$ is also an instance of the same axiom. If $\vdash_{n+1} x: \sim A, \Gamma \Rightarrow \Delta$, then if it is obtained by $(\mathrm{L} \sim)$ with $x: \sim A$ principal, i.e. it is of the form

$$
\frac{\vdash_{n} x S z, \Gamma \Rightarrow \Delta, z: A}{\vdash_{n+1} x: \sim A, \Gamma \Rightarrow \Delta}(\mathrm{L} \sim)
$$

where $z$ does not occur in the conclusion; then by dp-admissible substitution, $\vdash_{n} x S y, \Gamma \Rightarrow \Delta, y: A$ (where $y$ is a fresh variable).

We exemplify with $(\mathrm{R} \rightarrow)$ the case where $x: \sim A$ is obtained by a rule with eigenvalue condition in which it is not principal

$$
\frac{\vdash_{n} z \leq t, t: C, x: \sim A, \Gamma^{\prime} \Rightarrow \Delta^{\prime}, t: D}{\vdash_{n+1} x: \sim A, \Gamma^{\prime} \Rightarrow \Delta^{\prime}, z: C \rightarrow D}(\mathrm{R} \rightarrow)
$$

then by dp-substitution, $\vdash_{n} z \leq t^{\prime}, t^{\prime}: C, x: \sim A, \Gamma^{\prime} \Rightarrow \Delta^{\prime}, t^{\prime}: D$, where $t^{\prime} \not \equiv$ $y$. (Note that $y$ is fixed beforehand.) By I.H., $\vdash_{n} z \leq t^{\prime}, t^{\prime}: C, x S y, \Gamma^{\prime} \Rightarrow$ $\Delta^{\prime}, y: A, t^{\prime}: D$. So by $(\mathrm{R} \rightarrow), \vdash_{n+1} x S y, \Gamma^{\prime} \Rightarrow \Delta^{\prime}, y: A, z: C \rightarrow D$. 
If it is obtained by a rule without eigenvariable condition, then apply I.H. to the premise and apply the same rule.

Proposition 3.9 (dp-admissibility of Contraction). (LC) and (RC) are dp-admissible in $\mathbf{G 3 C c}_{\omega}^{+}$.

Proof: We argue by simultaneous induction ((LC),(RC)) on the depth of the deduction. General outline is as in [8, Theorem 12.28]. As an example, suppose $\vdash_{n+1} x: \sim A, x: \sim A, \Gamma \Rightarrow \Delta$ and the last step is an instance of $(\mathrm{L} \sim)$ with $x: \sim A$ principal.

$$
\frac{\vdash_{n} x S y, x: \sim A, \Gamma \Rightarrow \Delta, y: A}{\vdash_{n+1} x: \sim A, x: \sim A, \Gamma \Rightarrow \Delta}(\mathrm{L} \sim)
$$

Then by dp-admissible invertibility of $(\mathrm{L} \sim)$,

$$
\vdash_{n} x S y, x S y, \Gamma \Rightarrow \Delta, y: A, y: A
$$

So by I.H.

$$
\vdash_{n} x S y, \Gamma \Rightarrow \Delta, y: A
$$

Thus by $(\mathrm{L} \sim)$

$$
\vdash_{n+1} x: \sim A, \Gamma \Rightarrow \Delta
$$

We are now ready to prove the admissibility of (Cut). We shall call the item to be eliminated in (Cut) the cut-item.

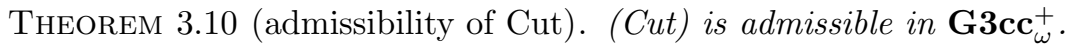

Proof: We argue by induction on the complexity of cut-items, with a subinduction on the level (the sum of the depths of the deductions of the premises) of (Cut). Again the outline is the same as that of the intuitionistic case [8, Theorem 12.30]. In particular, rules that are mathematical or geometric are treated similarly to those of intermediate axioms.

Here we shall consider the case where the cut-item is principal in both of the premises, and has the form $x: \sim A$. We have

$$
\frac{\vdash_{m-1} x S y, y: A, \Gamma \Rightarrow \Delta, x: \sim A}{\frac{\vdash_{m} x S y, \Gamma \Rightarrow \Delta, x: \sim A}{\vdash}(\mathrm{R} \sim) \quad \frac{\vdash_{n-1} x S z, \Gamma^{\prime} \Rightarrow \Delta^{\prime}, z: A}{\vdash_{n} x: \sim A, \Gamma^{\prime} \Rightarrow \Delta^{\prime}}(\mathrm{L} \sim)} \text { (Cut) }
$$


Then

$$
\begin{aligned}
& \vdash_{n-1} x S z, \Gamma^{\prime} \Rightarrow \Delta^{\prime}, z: A \\
& \vdash_{n-1} x \bar{S} \bar{y}, \bar{\Gamma}^{\prime} \Rightarrow \bar{\Delta}^{\prime}, \bar{y}: \bar{A}
\end{aligned}
$$

(note that $z$ is an eigenvariable, so cannot occur in $\Gamma^{\prime}, \Delta^{\prime}$ ). Moreover, by I.H. the following cut of a lower level $(m+n-1<m+n)$ is admissible:

$$
\begin{aligned}
\vdash_{m_{-1}} x S y, y: A, \Gamma \Rightarrow \Delta, x: \sim A & \vdash_{n} x: \sim A, \Gamma^{\prime} \Rightarrow \Delta^{\prime} \\
& \vdash x \overline{S y}, y: \bar{A}, \overline{\Gamma \Gamma^{\prime}} \Rightarrow \Delta^{\Delta^{\prime}}
\end{aligned}
$$

From these, and a cut of lower complexity (admissible by I.H.), we obtain

$$
\begin{aligned}
& \vdash_{n-1} x S y, \Gamma^{\prime} \Rightarrow \Delta^{\prime}, y: A \quad \vdash x S y, y: A, \Gamma \Gamma^{\prime} \Rightarrow \Delta \Delta^{\prime} \\
& \begin{array}{l}
\vdash x \bar{S} y, x \bar{S} y, \bar{\Gamma} \Gamma^{\prime} \Gamma^{\prime} \Rightarrow \bar{\Delta} \bar{\Delta}^{\prime} \Delta^{\prime}-\text { (Contraction) } \\
\qquad \bar{\vdash} \bar{S} y, \bar{\Gamma} \Gamma^{\prime} \Rightarrow \Delta \Delta^{\prime}
\end{array}
\end{aligned}
$$

Next we observe that $\mathbf{G} 3 \mathbf{c c} \mathbf{c}_{\omega}$ and calculi in $\mathbf{G} 3 \mathbf{c c}_{\omega}^{+}$indeed correspond to $\mathbf{C C}_{\omega}$, daC, $\mathbf{T} \mathbf{C C}_{\omega}$ and $\mathbf{I P C}{ }^{\sim}$.

Proposition 3.11.

(i) $\vdash_{c} A$ implies $\vdash_{G c} \Rightarrow x: A$.

(ii) If we add $(\mathrm{Pr}) /\left(\operatorname{trans}_{S}\right)$ to the calculus, then the axioms of $\mathbf{d a C} / \mathbf{T C C} \mathbf{C}_{\omega}$ become derivable. If we add $\left(\operatorname{trans}_{S}\right)$ and $(\mathrm{De})$, the axioms of $\mathbf{I P C}^{\sim}$ become derivable.

\section{Proof:}

(i) For $\mathbf{C C}_{\omega}$, the positive axioms can be shown to be derivable as in the intuitionistic case. We need to check $[\mathrm{Ax} 9],[\mathrm{Ax} 10]$ and $[\mathrm{RC}]$.

$[\mathrm{Ax} 9]$

$$
\begin{gathered}
\frac{x \leq x, x S x, x: A \Rightarrow x: A, x: \sim A}{x S x, x: A \Rightarrow x: A, x: \sim A}(\mathrm{Ref}) \\
\frac{x S x \Rightarrow x: A, x: \sim A}{\Rightarrow}\left(\operatorname{Ref}_{S}\right) \\
\frac{\Rightarrow x: A, x: \sim A}{\Rightarrow x: A \vee \sim A}(\mathrm{R} \vee)
\end{gathered}
$$


$\underline{[\mathrm{Ax} 10]}$

$$
\begin{aligned}
& \frac{y \leq y, x \leq y, y S z, z S y, y: A \Rightarrow z: \sim A, y: A}{x \leq y, y S z, z S y, y: A \Rightarrow z: \sim A, y: A} \text { (Ref) } \\
& \frac{x \leq y, y S z, z S y \Rightarrow z: \sim A, y: A}{x \leq y, y S z \Rightarrow z: \sim A, y: A}\left(\mathrm{Sym}_{S}\right) \\
& \frac{x \leq y, y: \sim \sim A \Rightarrow y: A}{\Rightarrow x: \sim \sim A \rightarrow A}(\mathrm{R} \rightarrow)
\end{aligned}
$$

$\underline{\mathrm{RC}]}$

We first observe that $x: A \rightarrow B, x: A \Rightarrow x: B$ is derivable:

$$
\frac{x \leq x, x: A \rightarrow B, x: A \Rightarrow x: A \quad x \leq x, x: A \rightarrow B, x: B \Rightarrow x: B}{\frac{x \leq x, x: A \rightarrow B, x: A \Rightarrow x: B}{x: A \rightarrow B, x: A \Rightarrow x: B}(\operatorname{Ref})}(\mathrm{L} \rightarrow)
$$

Then

$$
\begin{aligned}
& \Rightarrow x: A \rightarrow B \quad x: A \rightarrow B, x: A \Rightarrow x: B \quad(\mathrm{Cut}) \\
& \bar{y} \leq \bar{z}, \overline{S S} \bar{x}, \bar{x}: \bar{A} \Rightarrow \bar{x}: \bar{B}, \bar{z}: \overline{\sim A} \text { (Weakening) } \\
& \frac{y \leq z, z S x \Rightarrow x: B, z: \sim A}{y \leq z, z: \sim B \Rightarrow z: \sim A}(\mathrm{~L} \sim) \\
& \begin{array}{l}
\leq z, z: \sim B \Rightarrow z: \sim A \\
\Rightarrow y: \sim B \rightarrow \sim A \\
\Rightarrow-\bar{S} \rightarrow(\mathrm{Rub}) \\
\Rightarrow x: \sim \bar{B} \rightarrow \bar{\sim}
\end{array}
\end{aligned}
$$

(ii) We need to check each of the additional axioms are derivable in the corresponding calculi.

\section{daC}

First we apply (LV) to

$$
\begin{aligned}
& x \leq y, y S z, z S t, t \leq y, t \leq z, t: A \Rightarrow z: \sim(A \vee B), z: A, y: B \\
& x \leq y, y S z, z S t, t \leq y, t \leq z, t: B \Rightarrow z: \sim(A \vee B), z: A, y: B
\end{aligned}
$$


to obtain

$$
x \leq y, y S z, z S t, t \leq y, t \leq z, t: A \vee B \Rightarrow z: \sim(A \vee B), z: A, y: B
$$

Then,

$$
\begin{gathered}
x \leq y, y S z, z S t, t \leq y, t \leq z, t: A \vee B \Rightarrow z: \sim(A \vee B), z: A, y: B \\
\frac{x \leq y, y S z, z S t, t \leq y, t \leq z \Rightarrow z: \sim(A \vee B), z: A, y: B}{\frac{x \leq y, y S z \Rightarrow z: \sim(A \vee B), z: A, y: B}{x \leq y, y S z \Rightarrow z: \sim(A \vee B) \vee A, y: B}}(\mathrm{R} \vee) \\
\frac{\frac{x}{x \leq y, y: \sim(\sim(A \vee B) \vee A) \Rightarrow y: B}}{\Rightarrow x: \sim(\sim(A \vee B) \vee \sim A) \rightarrow B}(\mathrm{~L}) \\
(\mathrm{R} \rightarrow)
\end{gathered}(\mathrm{R})
$$

$\underline{\mathrm{TCC}_{\omega}}$

$$
\begin{aligned}
& \begin{array}{c}
u \leq u, y \leq z, y S u, z S v, z S u, v S z, v S u, u: A \Rightarrow u: A, v: \sim A \\
\frac{y \leq z, y S u, z S v, z S u, v S z, v S u, u: A \Rightarrow u: A, v: \sim A}{y \leq z, y S u, z S v, z S u, v S z, v S u \Rightarrow u: A, v: \sim A} \text { (Ref) }
\end{array} \\
& y \leq z, y S u, z S v, z S u, v S z \Rightarrow u: A, v: \sim A\left(\operatorname{Trans}_{S}\right) \\
& y \leq z, y S u, z S v, z S u \Rightarrow u: A, v: \sim A(\mathrm{Up}) \\
& \frac{y \leq z, y S u, z S v \Rightarrow u: A, v: \sim A}{y \leq z, y S u, z: \sim \sim A \Rightarrow u: A}(\mathrm{~L} \sim) \\
& y \leq z, y: \sim A, z: \sim \sim A \Rightarrow \quad(\mathrm{L} \sim) \\
& \bar{x} \leq \bar{y}, \bar{y} \leq \bar{z}, y: \bar{\sim}, \bar{z}: \bar{\sim} \bar{A} \Rightarrow \bar{z}: \bar{B} \text { (Weakening) } \\
& \frac{x \leq y, y: \sim A \Rightarrow y: \sim \sim A \rightarrow B}{x: \sim A \rightarrow(\sim \sim A \rightarrow B)}(\mathrm{R} \rightarrow)
\end{aligned}
$$

\section{$\underline{\text { IPC }^{\sim}}$}

First we apply ( $\mathrm{LV})$ to

$$
\begin{aligned}
& y S z, y S t, w: A, w \leq z, w \leq t, y S w \Rightarrow z: A, t: B, y: \sim(A \vee B) \\
& y S z, y S t, w: B, w \leq z, w \leq t, y S w \Rightarrow z: A, t: B, y: \sim(A \vee B)
\end{aligned}
$$

to obtain

$$
y S z, y S t, w: A \vee B, w \leq z, w \leq t, y S w \Rightarrow z: A, t: B, y: \sim(A \vee B)
$$


Then

$$
\begin{aligned}
& \frac{y S z, y S t, w: A \vee B, w \leq z, w \leq t, y S w \Rightarrow z: A, t: B, y: \sim(A \vee B)}{y S z, y S t, w \leq z, w \leq t, y S w \Rightarrow z: A, t: B, y: \sim(A \vee B)}(\mathrm{R} \sim) \\
& \frac{y S z, y S t \Rightarrow z: A, t: B, y: \sim(A \vee B)}{y S z, y: \sim B \Rightarrow z: A, y: \sim(A \vee B)}(\mathrm{L} \sim) \\
& \frac{y S z, y: \sim B \Rightarrow z: A, y: \sim(A \vee B)}{y: \sim A, y: \sim B \Rightarrow y: \sim(A \vee B)}(\mathrm{L} \sim) \\
& y: \sim A \wedge \sim B \Rightarrow y: \sim(A \vee B)(\mathrm{L} \wedge) \\
& \bar{x} \leq y, \bar{y}: \bar{A} \wedge \sim \bar{B} \Rightarrow y: \bar{\sim} \bar{A} \vee \bar{B}) \text { (Weakening) } \\
& \Rightarrow x:(\sim A \wedge \sim B) \rightarrow \sim(A \vee B) \quad(\mathrm{R} \rightarrow)
\end{aligned}
$$

For the converse direction, we argue via completeness of the Hilbertstyle systems with respect to Kripke semantics. For this purpose the notion of valuation has to be modified in the style of [8, Definition 11.25-26], to accommodate labelled formulae and relational atoms.

Definition 3.12 (modified valuation for $\mathbf{C C}_{\omega}$-model). Let $\mathcal{F}_{\mathcal{K}}^{c}$ be a $\mathbf{C C}_{\omega^{-}}$ frame. A modified valuation $\mathcal{V}_{m}$ is a pair $(\mathcal{V}, l)$, where $\mathcal{V}$ is the ordinary valuation for $\mathcal{F}_{\mathcal{K}}^{c}$ introduced before, and $l$ maps each label $x$ into to a world $l(x)$ of $\mathcal{F}_{\mathcal{K}}^{c}$.

We say an item $\alpha$ is valid in a modified model $\mathcal{M}_{\mathcal{K}}^{m c}=\left(\mathcal{F}_{\mathcal{K}}^{c}, \mathcal{V}_{m}\right)($ denoted $\left.\mathcal{M}_{\mathcal{K}}^{m c} \Vdash_{\mathcal{K} c}^{m} \alpha\right)$, when

- $l(x) \leq l(y)($ or $l(x) S l(y))$ in $\mathcal{F}_{\mathcal{K}}^{c}$, if $\alpha \equiv x \leq y$ (or $\left.x S y\right)$.

- $\left(\mathcal{F}_{\mathcal{K}}^{c}, \mathcal{V}\right), l(x) \Vdash_{\mathcal{K}_{c}} A$, if $\alpha \equiv x: A$.

We say a sequent $\Gamma \Rightarrow \Delta$ is valid in $\mathcal{M}_{\mathcal{K}}^{m c}\left(\right.$ denoted $\left.\mathcal{M}_{\mathcal{K}}^{m c} \models_{\mathcal{K}_{c}}^{m} \Gamma \Rightarrow \Delta\right)$, if $\mathcal{M}_{\mathcal{K}}^{m c} \Vdash_{\mathcal{K} c}^{m} \alpha$ for all $\alpha \in \Gamma$ implies $\mathcal{M}_{\mathcal{K}}^{m c} \Vdash_{\mathcal{K}^{c}}^{m} \beta$ for some $\beta \in \Delta$. If $\mathcal{M}_{\mathcal{K}}^{m c}$ is arbitrary, we say $\Gamma \Rightarrow \Delta$ is valid and write $\vDash_{\mathcal{K}_{c}}^{m} \Gamma \Rightarrow \Delta$.

Note that $\models_{\mathcal{K}_{c}}^{m} \Rightarrow x: A$ is valid if and only if $\vDash_{\mathcal{K}_{c}} A$. Similar statements hold when we restrict the class of frames. We now wish to demonstrate the following.

Proposition 3.13.

(i) $\vdash_{G c} \Gamma \Rightarrow \Delta$ implies $\vDash_{\mathcal{K}_{c}}^{m} \Gamma \Rightarrow \Delta$.

(ii) $(\operatorname{Pr}) /\left(\operatorname{Trans}_{S}\right) /(\mathrm{De})$ become sound if we restrict consideration to the corresponding classes of frames. 


\section{ProOF:}

(i) We argue by induction on the depth of deductions. The cases for the intuitionistic rules follow straightforwardly from the definition of intuitionistic Kripke models. We shall look at the cases for $(\mathrm{L} \sim),(\mathrm{R} \sim)$. The other cases are straightforward. In each case, we consider an arbitrary modified model $\mathcal{M}_{\mathcal{K}}^{m c}=\left(\mathcal{F}_{\mathcal{K}}^{c}, \mathcal{V}_{m}\right)$ with $\mathcal{V}_{m}=(\mathcal{V}, l)$.

$(\mathrm{L} \sim)$ Suppose $\mathcal{M}_{\mathcal{K}}^{m c} \Vdash_{\mathcal{K}_{c}}^{m} \alpha$ for all $\alpha \in\{x: \sim A\} \cup \Gamma$. Then $\left(\mathcal{F}_{\mathcal{K}}^{c}, \mathcal{V}\right), l(x) \Vdash_{\mathcal{K}_{c}}$ $\sim A$. So there is $w$ such that $l(x) S w$ and $\left(\mathcal{F}_{\mathcal{K}}^{c}, \mathcal{V}\right), w \nVdash_{\mathcal{K}_{c}} A$. Take $\mathcal{V}_{m}^{\prime}=\left(\mathcal{V}, l^{\prime}\right)$ where $l^{\prime}=l$ except $l^{\prime}(y)=w$. Note, since $y$ does not occur in $\Gamma$ and $\Delta, l$ and $l^{\prime}$ evaluate them in the same way. Thus $\left(\mathcal{F}_{\mathcal{K}}^{c}, \mathcal{V}_{m}^{\prime}\right) \Vdash_{\mathcal{K}_{c}}^{m} \alpha$ for all $\alpha \in\{x S y\} \cup \Gamma$. So by I.H., $\left(\mathcal{F}_{\mathcal{K}}^{c}, \mathcal{V}_{m}^{\prime}\right) \Vdash_{\mathcal{K}_{c}}^{m} \beta$ for some $\beta \in\{y: A\} \cup \Delta$. If it validates $y: A$, however, then $\left(\mathcal{F}_{\mathcal{K}}^{c}, \mathcal{V}\right), l^{\prime}(y) \Vdash_{\mathcal{K}_{c}} A$, a contradiction. Therefore $\left(\mathcal{F}_{\mathcal{K}}^{c}, \mathcal{V}_{m}\right) \Vdash_{\mathcal{K} c}^{m} \beta$ for some $\beta \in \Delta$. Since $\mathcal{M}_{\mathcal{K}}^{m c}$ is arbitrary, $\vDash_{\mathcal{K}_{c}}^{m} x: \sim A, \Gamma \Rightarrow \Delta$.

$(\mathrm{R} \sim)$ Suppose $\mathcal{M}_{\mathcal{K}}^{m c} \Vdash_{\mathcal{K} c}^{m} \alpha$ for all $\alpha \in\{x S y\} \cup \Gamma$. If $l(y) \nVdash_{\mathcal{K}_{c}} A$, then $l(x) \Vdash_{\mathcal{K}_{c}} \sim A$. Otherwise, $l(y) \Vdash_{\mathcal{K}_{c}} A$, so by I.H., $\mathcal{M}_{\mathcal{K}}^{m c} \Vdash_{\mathcal{K}_{c}}^{m} \alpha$ for all $\alpha \in\{x S y, y: A\} \cup \Gamma$. So in either case (the latter with I.H.), $\mathcal{M}_{\mathcal{K}}^{m c} \Vdash_{\mathcal{K} c}^{m} \beta$ for some $\beta \in \Delta \cup\{x: \sim A\}$.

(ii) The case for $\left(\operatorname{Trans}_{S}\right)$ is straightforward and (Pr), (De) are similar to the case for $(\mathrm{L} \sim)$; one needs to appeal to the frame condition to pick out a world satisfying the desired order relation; then define a new modified valuation which is identical to the original except it assigns the world to the eigenvariable; then the rest follows as in the case for $(\mathrm{L} \sim)$.

This allows us to conclude the other direction.

Corollary 3.14 .

(i) $\vdash_{G c} \Rightarrow x: A$ implies $\vdash_{c} A$.

(ii) $\vdash_{G c+}$ is sound with respect to the corresponding logics $\left(\mathbf{d a C}, \mathbf{T C C}_{\omega}, \mathbf{I P C}^{\sim}\right)$.

ProOF:

(i) If $\vdash_{G c} \Rightarrow x: A$, then by the previous proposition, $\models_{\mathcal{K}_{c}}^{m} \Rightarrow x: A$. Then as we remarked before, $\vDash_{\mathcal{K}_{c}} A$. Thus by the completeness of $\mathbf{C C}_{\omega}, \vdash_{c} A$.

(ii) Similar. 


\section{Discussion}

We have seen that $\mathbf{T} \mathbf{C C}_{\omega}$ can be regarded as the logic of empirical and conegation for Beth semantics, which differs from $\mathbf{I P C}{ }^{\sim}$ and $\mathbf{d a C}$ for Kripke semantics. According to the interpretation in [11, p.679], the difference between Kripke and Beth semantics is the treatment of time. A node in Kripke models signifies a state of information, whereas in Beth models it signifies a moment in time. So for instance, to decide the forcing of a disjunction in a Kripke model, one can stay in a world as much as one likes, until one learns which of the disjuncts is true. In comparison, in Beth models this waiting time is expressed by posterior nodes, so we need to refer to those other worlds to decide the forcing of the disjunction in the original world. The two kinds of empirical and co-negation can be interpreted similarly.

The question remains, however, which empirical (or co- ) negation one actually means in an assertion of negation. For example, if one says "There is no proof of $\mathrm{P}=\mathrm{NP}$ ", does it mean there is no proof at the present state of information, or there is no proof at the present moment?

Changing perspective, from an intuitionistic viewpoint there is a certain advantage in considering Beth semantics. There is a relatively simple proof of intuitionistic completeness (proving completeness with only intuitionistically accepted principles) for intuitionistic logic $[3,11]$. The intuitionistic completeness proof for Kripke semantics [12] gives a more refined result, but is comparatively more involved. A possible future direction is to show the intuitionistic completeness for $\mathbf{T} \mathbf{C} \mathbf{C}_{\omega}$. An obstacle would be the treatment of excluded middle, but classical logic also has an intuitionistic completeness proof [5], so possibly this may be overcome. An intuitionistic completeness would be desirable if one is a full-fledged intuitionist, especially when the logic is motivated from the semantics, rather than from the syntax.

Acknowledgements. This research was supported by the Japan Society for the Promotion of Science (JSPS), Core-to-Core Program (A. Advanced Research Networks). The author is indebted to Hitoshi Omori and Giulio Fellin for their suggestion to look at empirical negation and co-negation, respectably. He also thanks Hajime Ishihara, Takako Nemoto and Keita Yokoyama for their encouragement and many valuable suggestions during 
the production of this paper. Lastly, he thanks the anonymous reviewer for the helpful comments and suggestions.

\section{References}

[1] M. De, Empirical Negation, Acta Analytica, vol. 28 (2013), pp. 49-69, DOI: http://dx.doi.org/10.1007/s12136-011-0138-9.

[2] M. De, H. Omori, More on Empirical Negation, [in:] R. Goreé, B. Kooi, A. Kurucz (eds.), Advances in Modal Logic, vol. 10, College Publications (2014), pp. 114-133.

[3] H. Friedman, Intuitionistic Completeness of Heyting's Predicate Calculus, Notices of the American Mathematical Society, vol. 22(6) (1975), pp. A648-A648.

[4] A. B. Gordienko, A Paraconsistent Extension of Sylvan's Logic, Algebra and Logic, vol. 46(5) (2007), pp. 289-296, DOI: http://dx.doi.org/10.1007/ s10469-007-0029-8.

[5] V. N. Krivtsov, An intuitionistic completeness theorem for classical predicate logic, Studia Logica, vol. 96(1) (2010), pp. 109-115, DOI: http://dx.doi. org/10.1007/s11225-010-9273-3.

[6] S. Negri, Proof analysis in modal logic, Journal of Philosophical Logic, vol. 34(5-6) (2005), pp. 507-544, DOI: http://dx.doi.org/10.1007/s10992005-2267-3.

[7] S. Negri, Proof analysis in non-classical logics, [in:] C. Dimitracopoulos, L. Newelski, D. Normann, J. Steel (eds.), ASL Lecture Notes in Logic, vol. 28, Cambridge University Press (2007), pp. 107-128, DOI: http://dx. doi.org/10.1017/CBO9780511546464.010.

[8] S. Negri, J. von Plato, Proof analysis: a contribution to Hilbert's last problem, Cambridge University Press (2011), DOI: http://dx.doi.org/10. 1017/CBO9781139003513.

[9] G. Priest, Dualising Intuitionistic Negation, Principia, vol. 13(2) (2009), pp. 165-184, DOI: http://dx.doi.org/10.5007/1808-1711.2009v13n2p165.

[10] R. Sylvan, Variations on da Costa C Systems and dual-intuitionistic logics I. Analyses of $C_{\omega}$ and $C C_{\omega}$, Studia Logica, vol. 49(1) (1990), pp. 47-65, DOI: http://dx.doi.org/10.1007/BF00401553. 
Empirical Negation, Co-negation and the Contraposition Rule II. . 375

[11] A. S. Troelstra, D. van Dalen, Constructivism in Mathematics: An Introduction, vol. II, Elsevier (1988).

[12] W. Veldman, An Intuitionistic Completeness Theorem for Intuitionistic Predicate Logic, The Journal of Symbolic Logic, vol. 41(1) (1976), pp. 159-166, DOI: http://dx.doi.org/10.2307/2272955.

\section{Satoru Niki}

Japan Advanced Institute of Science and Technology

School of Information Science

923-1292, 1-1 Asahidai, Nomi

Ishikawa, Japan

e-mail: satoruniki@jaist.ac.jp 